\title{
Ultrasonographic study of the right coronary artery in performance horses
}

\author{
Cristóbal A. Dörner ${ }^{\text {abc*, Constanza Chavarría }}{ }^{a}$
}

\begin{abstract}
Historically, echocardiography has focused on the assessment of cardiac dimensions and indices of cardiac function. However, when referred to ultrasonographic coronary arteries characterisation in the horse, information is scarce. The purpose of this study was to describe the right coronary artery in both long and short axis and to analyse its variability between horses with different levels of performance. Sixty healthy horses were included in the study. The animals were eventing horses at different levels of performance and were allocated into two groups according to their athletic level. The internal lumen diameter and the area of the right coronary artery (RCA) were measured in the right parasternal long and short axis views in the 3rd and 4th intercostal space respectively, during systole and diastole. The results were compared between groups using the analysis of variance (ANOVA) and Student's t-test. The correlation between the physiological parameters and the RCA was assessed using the Pearson correlation coefficient. Statistically significant differences were found when the RCA internal diameter was assessed between groups in both short and long axis. There were no statistically significant differences intra-group when short and long axis measurements were compared between each other. A positive correlation was observed between RCA and age, height, left atrium diameter, aortic valve diameter, sinus of Valsalva diameter, and aorta diameter. In conclusion, the level of training might affect the ultrasound measurement of the RCA in sport horses. Non-invasive echocardiography can be used to consistently assess the size of the right coronary artery in athletic horses in both short and long axis.

Key words: coronary artery, echocardiographic, heart, horses, eventing.
\end{abstract}

\section{INTRODUCTION}

The heart is a complex and fascinating organ with a variety of physiological and anatomical properties that aim to pump blood to the body. To make this possible, the heart muscle and its inner structures need to be well irrigated and oxygenated. The blood is distributed through the myocardium thanks to two coronary arteries arising from the right and left sinus of Valsalva (Bright et al 2010, Marr and Patteson 2010).

Echocardiography, including two-dimensional (2D), M-mode and various Doppler modalities, have revolutionised equine cardiology. These technologies provide a better understanding of the normal physiology and morphology of the equine heart and an improved ability to diagnose and assess the severity of many forms of heart disease, especially in performance horses. Historically, echocardiography has centred on the assessment of cardiac dimensions and indices of cardiac function. However, when referred to ultrasonographic coronary arteries characterisation in the horse, information is scarce and to our knowledge only one recent pilot study has been published (Siwinska et al 2019).

Giving the importance of myocardial irrigation in the normal function of the heart, the evaluation of the coronary arteries in selected cases could be considered

Received: 07.10.2020.

Accepted: 11.05.2021.

${ }^{a}$ Escuela de Medicina Veterinaria, Facultad de Recursos Naturales y Medicina Veterinaria, Universidad Santo Tomás, Viña del Mar, Chile.

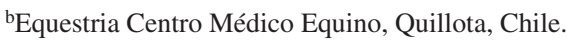

${ }^{c}$ Campo Militar San Isidro, Ejército de Chile, Quillota, Chile.

*Corresponding author: CA Dörner; Avenida Larraguibel S/N, San Isidro, Quillota, Chile; cdorner@gmail.com during cardiac evaluation, as routinely done in humans (Dodge et al 1992, Arnoudse et al 2007, Kumar Raut et al 2017). Anatomical and morphological studies of the heart in different species have shown the predominance of the right coronary artery (Gómez et al 2017) and its ultrasonographic dimension was recently studied in normal horses ( $\mathrm{n}=36$ ) (Siwinska et al 2019). The ultrasonographic characterisation of the right coronary artery in the horse is performed in the right parasternal window, long axis at the level of the right outflow tract (Siwinska et al 2019). Nonetheless, the technique and normal ultrasound image of the right coronary artery in short axis at the level of the sinus of Valsalva has not been described.

This study aimed to describe the right coronary artery in both long and short axis in the right parasternal window, evaluate its presence repeatability during cardiac evaluation, evaluate if there are any differences in the diameter and/ or area when the artery is measured in different axis intragroup, and finally to analyse its variability between horses of different levels of performance.

\section{MATERIAL AND METHODS}

Sixty eventing performance horses (21 mares, 39 geldings), clinically healthy and competing at their intended level of performance, aged 5-16 years (mean $8.33 \pm 2.589$ years old), weighing 495-569 kg with a wither's height of $159-168 \mathrm{~cm}$ were included in the study. All horses were Warmblood horses (Holsteiner, Selle Français and Holsteiner $x$ Selle Francais crossbred). A complete physical examination and an echocardiographic examination were performed of each animal included in the study. The exclusion criteria were as follows: systemic illness, dysrhythmia (excluding a physiological second-degree atrioventricular block), 
audible heart murmurs or any heart abnormality found during echocardiography.

The animals were divided into two groups according to their level of performance. Group I contained 30 adult horses ranging from 5 to 8 years old and they were starting at eventing or were competing at $\mathrm{CCI} 1 *$ according to the categorisation provided by the Fédération Équestre Internationale (2020). Group II contained 30 adult animals from 8 to 16 years old that regularly participated in $\mathrm{CC} 2 *_{-} \mathrm{S}$ to $\mathrm{CCI} 4 * \mathrm{~L}$ level, according to the same categorisation. All horses in both groups had undergone regular training for at least one year.

Echocardiography was performed using an Mindray ${ }^{\circledR}$ M-5 ultrasound scanner, equipped with a phased array 2.5-5.0MHz transducer. Prior to echocardiography, the right parasternal window was clipped and the site was washed with chlorhexidine-based soap and warm water. Isopropyl alcohol was then applied as a coupler between the transducer and the skin. Two-dimensional (B-Mode) and M-Mode imaging were conducted at an imaging depth of $30 \mathrm{~cm}$ from the right hemithorax. To obtain the left ventricle (LV) measurements, M-Mode was used at the level of papillary muscles. Heart rate (HR), Interventricular septal thickness at the end-diastole and systole (IVSd and IVSs respectively), LV internal diameter (LVIDd and LVIDs), LV free wall thickness (LVFWd and LVFWs) were measured using the ultrasound calliper. Using Teichholz's formula included in the scanner's software, LV fractional shortening (FS), end diastolic volume (EDV), end systolic volume (ESV), LV ejection fraction $(\mathrm{EF})$, stroke volume $(\mathrm{SV})$ and cardiac output (CO) were calculated. Measurements of the RCA were done in B-Mode on both the short and long axis. The right coronary artery lumen was measured in the long axis at the 3 rd intercostal space at the right outflow tract (figure 1). The coronary artery was also measured in a short axis at the 4th intercostal space at the level of the aortic sinus of Valsalva (figure 2).

Statistical analyses were run on SPSS, version 19 for Windows (SPSS Inc, Chicago IL, USA). KolmogorovSmirnov test was used to determine the normal distribution of the data. ANOVA was run to determine statistical differences between groups on both the long and short axis. A Pearson correlation test was run to determine the association between the RCA and the Two-dimensional (BMode) and M-Mode cardiac measurements. Additionally, a paired sample T-test was used to compare the RCA measurements obtained between the long and short axis. The significance level was set at $P<0.05$.

\section{RESULTS}

All data were normally distributed. Echocardiographic parameters for both groups are presented in table 1. Group II showed greater measurements when compared with the horses allocated in group I, nonetheless significant differences $(P<0.05)$ were found only in the aortic valve, sinus of Valsalva and aorta.

Table 1. Mean values \pm and standard deviation (SD) of clinical data and measurements of the left ventricle study using two-dimensional and M-mode ultrasonography.

\begin{tabular}{|c|c|c|}
\hline Parameter & Group I & Group II \\
\hline Age (years) & $6.67 \pm 1.35$ & $10.1 \pm 2.46$ \\
\hline Heart Rate (bpm) & $42.1 \pm 14.06$ & $44.53 \pm 11.86$ \\
\hline LA diameter (mm) & $104.56 \pm 8.82$ & $111.82 \pm 12.47$ \\
\hline Aortic annulus (mm) & $54.40 \pm 6.55^{*}$ & $58.82 \pm 4.44 *$ \\
\hline Sinus of Valsalva (mm) & $78.32 \pm 5.32 *$ & $81.02 \pm 5.11 *$ \\
\hline Aorta diameter $(\mathrm{mm})$ & $59.74 \pm 6.57 *$ & $62.77 \pm 6.10^{*}$ \\
\hline LVIDs (mm) & $62.18 \pm 14.39$ & $62.09 \pm 12.48$ \\
\hline LVIDd (mm) & $109.09 \pm 14.60$ & $112.79 \pm 11.36$ \\
\hline IVSDs (mm) & $45.21 \pm 5.33$ & $47.86 \pm 5.37$ \\
\hline IVSDd (mm) & $27.59 \pm 3.28$ & $28.27 \pm 3.54$ \\
\hline LVFWs (mm) & $39.78 \pm 6.05$ & $38.83 \pm 6.41$ \\
\hline LVFWd (mm) & $22.67 \pm 5.08$ & $22.84 \pm 5.56$ \\
\hline $\mathrm{ESV}(\mathrm{mL})$ & $201.06 \pm 133.14$ & $205.49 \pm 105.65$ \\
\hline EDV (mL) & $697.16 \pm 221.16$ & $741.36 \pm 166.02$ \\
\hline FS $(\%)$ & $43.16 \pm 7.79$ & $45.12 \pm 7.11$ \\
\hline $\mathrm{EF}(\%)$ & $70.74 \pm 8.92$ & $73.14 \pm 8.06$ \\
\hline $\mathrm{SV}(\mathrm{mL})$ & $486.68 \pm 125.63$ & $536.55 \pm 104.57$ \\
\hline $\mathrm{CO}(\mathrm{L} / \mathrm{min})$ & $20.35 \pm 8.06$ & $24.25 \pm 9.33$ \\
\hline
\end{tabular}

*Statistically significant difference between groups $(P<0.05)$. 
In the right parasternal long axis view, the RCA was visible in the form of a centrally located circle with an anechogenic lumen surrounded by a hyperechogenic rim, located ventrally to the right atrium (RA), dorsally to the PA and laterally to the right ventricle (RV) (figure 1). In the right parasternal short axis view, the RCA was visible as parallel hyperechogenic lines with an anechogenic lumen emerging from the right sinus of Valsalva ventrally to the RA, dorsally to the left atrium (LA) and laterally to the Aorta (figure 2). The mean diameter of the RCA for horses in group I was $13.48 \pm 1.22 \mathrm{~mm}$ in systole and $10.87 \pm 0.94 \mathrm{~mm}$ in diastole when evaluated on the short axis and $13.5 \pm 1.46 \mathrm{~mm}$ in systole and $10.48 \pm 1.61 \mathrm{~mm}$ in diastole when evaluated on the long axis. The RCA calculated mean values for the short axis were $1.437 \pm$ $0.275 \mathrm{~cm}^{2}$ and $0.94 \pm 0.158 \mathrm{~cm}^{2}$ in systole and diastole respectively. The mean RCA area values for the long axis were $1.448 \pm 0.324 \mathrm{~cm}^{2}$ and $0.881 \pm 0.242 \mathrm{~cm}^{2}$ in systole

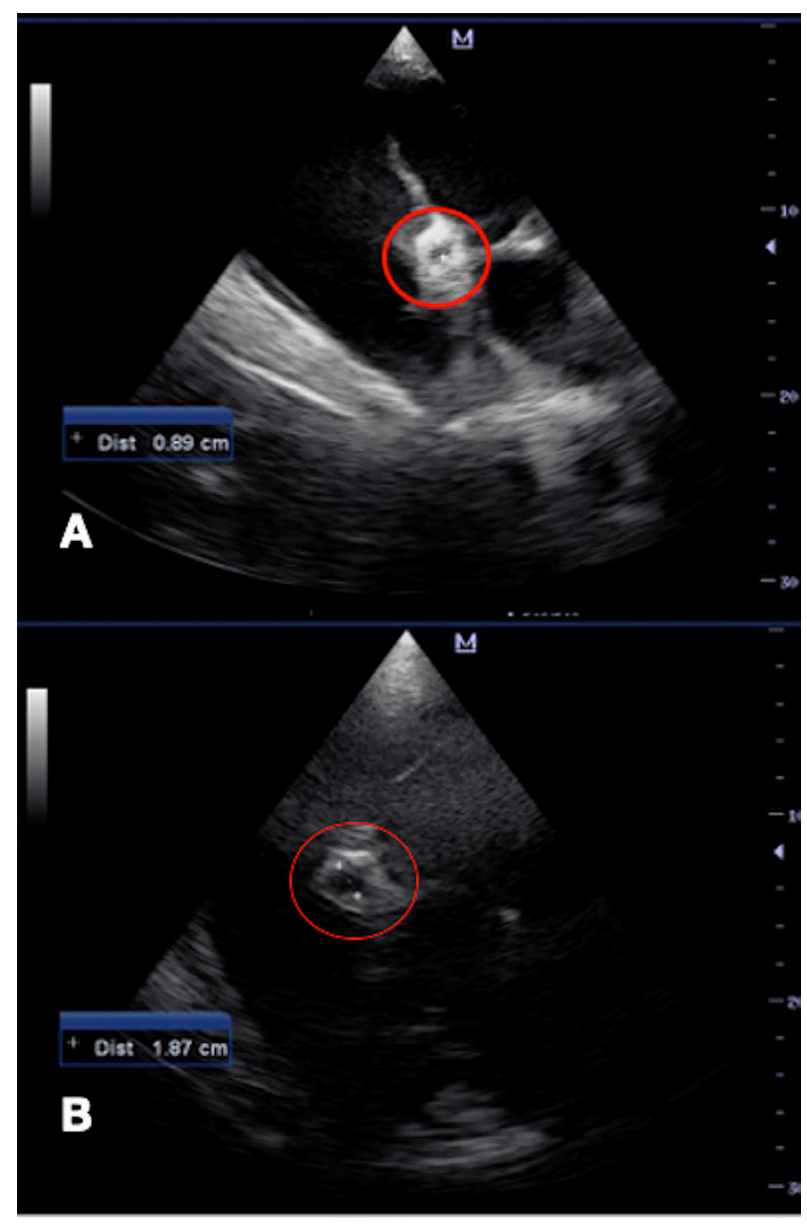

Figure 1. Right parasternal long axis ultrasonographic view of the right outflow tract showing the right coronary artery in diastole (A) and systole (B) as a round hyperechoic image with an anechoic lumen in the centre of the picture. A) Measure of the diameter using the machine calliper with the inner to inner method (red circle). Notice the diastole moment marked by an open tricuspid valve. B) systole marked by a closed tricuspid valve. and diastole, respectively. The mean diameter of the RCA for horses allocated in group II was $15.09 \pm 1.46 \mathrm{~mm}$ in systole and $11.91 \pm 1.22 \mathrm{~mm}$ in diastole when evaluated on the short axis and $15.44 \pm 1.84 \mathrm{~mm}$ in systole and 11.82 $\pm 1.45 \mathrm{~mm}$ in diastole when evaluated on the long axis. When the area was calculated, the mean values for the short axis were $1.805 \pm 0.364 \mathrm{~cm}^{2}$ and $1.126 \pm 0.228 \mathrm{~cm}^{2}$ in systole and diastole, respectively. For the long axis the mean values were $1.896 \pm 0.463 \mathrm{~cm}^{2}$ and $1.111 \pm 0.285$ $\mathrm{cm}^{2}$ in systole and diastole, respectively. ANOVA showed statistically significant differences $(P<0.05)$ between groups when the RCA diameter and area were analysed in both the short and long axis views at diastole and systole, respectively (table 2). There were no differences between the RCA measurements when compared between long and short axis views.

Pearson coefficient showed a positive correlation between the RCA measurements and age, height, LA diameter,

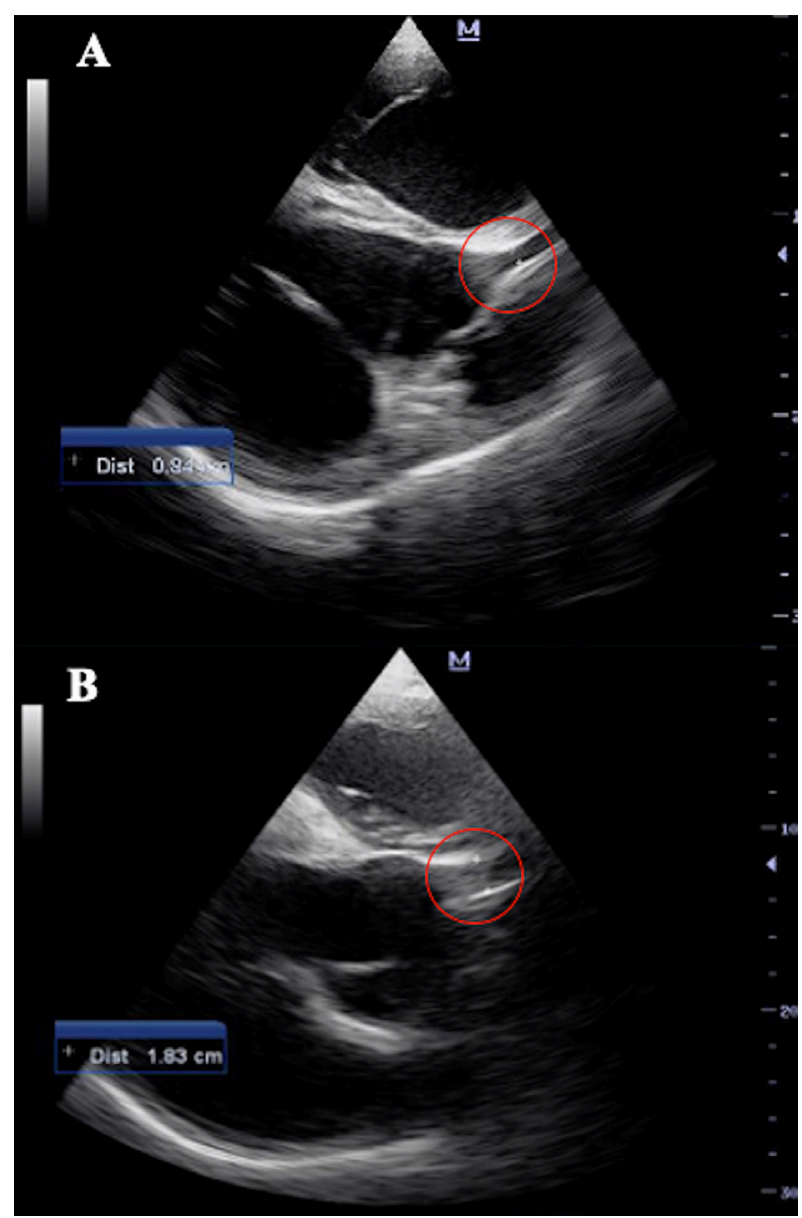

Figure 2. Same horse than figure 1. Right parasternal short axis ultrasonographic view at the level of the aorta (sinus of Valsalva) showing the right coronary artery in diastole (A) and systole (B) as a parallel hyperechoic line emerging from the right sinus of Valsalva. A) Measure of the diameter using the machine calliper with the inner to inner method (red circle). Notice the diastole moment marked by an open mitral valve and closed aortic valve. B) systole marked by an open aortic valve. 
DÖRNER, CHAVARRÍA

Table 2. Mean values \pm standard deviation $(\mathrm{SD})$ of the right coronary artery diameter and area obtained from two-dimensional echocardiography.

\begin{tabular}{lcc}
\hline Parameter & Group I & Group II \\
\hline \multicolumn{1}{c}{ Short axis } & & $15.09 \pm 1.46^{*}$ \\
RCAs diameter $(\mathrm{mm})$ & $13.48 \pm 1.22^{*}$ & $11.91 \pm 1.22^{*}$ \\
RCAd diameter $(\mathrm{mm})$ & $10.87 \pm 0.94^{*}$ & $1.805 \pm 0.364^{*}$ \\
RCAs area $\left(\mathrm{cm}^{2}\right)$ & $1.437 \pm 0.275^{*}$ & $1.126 \pm 0.228^{*}$ \\
RCAd area $\left(\left(\mathrm{cm}^{2}\right)\right.$ & $0.94 \pm 0.158^{*}$ & $15.44 \pm 1.84^{*}$ \\
$\quad$ Long axis & $13.50 \pm 1.46^{*}$ & $11.82 \pm 1.45^{*}$ \\
RCAs diameter $(\mathrm{mm})$ & $10.48 \pm 1.61^{*}$ & $1.896 \pm 0.463^{*}$ \\
RCAd diameter $(\mathrm{mm})$ & $1.448 \pm 0.324^{*}$ & $1.111 \pm 0.285^{*}$ \\
RCAs area $\left(\mathrm{cm}^{2}\right)$ & $0.881 \pm 0.242^{*}$ & \\
RCAd area $\left(\mathrm{cm}^{2}\right)$ & & \\
\hline
\end{tabular}

*Statistically significant difference between groups $(P<0.05)$.

Table 3. Correlation between RCA values and age, height, and left ventricle measurements.

\begin{tabular}{|c|c|c|c|c|c|c|c|c|}
\hline & \multicolumn{4}{|c|}{ Short axis } & \multicolumn{4}{|c|}{ Long axis } \\
\hline & $\begin{array}{c}\text { RCA } \\
\text { diameter } \\
\text { Systole }\end{array}$ & $\begin{array}{c}\text { RCA } \\
\text { diameter } \\
\text { Diastole }\end{array}$ & $\begin{array}{l}\text { RCA area } \\
\text { Systole }\end{array}$ & $\begin{array}{l}\text { RCA area } \\
\text { Diastole }\end{array}$ & $\begin{array}{l}\text { RCA diameter } \\
\text { Systole }\end{array}$ & $\begin{array}{c}\text { RCA } \\
\text { diameter } \\
\text { Diastole }\end{array}$ & $\begin{array}{l}\text { RCA area } \\
\text { Systole }\end{array}$ & $\begin{array}{c}\text { RCA area } \\
\text { Diastole }\end{array}$ \\
\hline Parameter & $\mathrm{r}$ & $\mathrm{r}$ & $\mathrm{r}$ & $\mathrm{r}$ & $\mathrm{r}$ & $\mathrm{r}$ & $\mathrm{r}$ & $\mathrm{r}$ \\
\hline Age & $0.594 * *$ & $0.470 * *$ & $0.585 * *$ & $0.461 * *$ & $0.472 * *$ & 0.244 & $0.459 * *$ & $0.282^{*}$ \\
\hline Height & $0.359 *$ & 0.260 & $0.363 * *$ & 0.268 & $0.377 * *$ & 0.272 & $0.388 * *$ & $0.184^{*}$ \\
\hline $\begin{array}{l}\text { LA diameter } \\
(\mathrm{mm})\end{array}$ & $0.412 * *$ & $0.504 * *$ & $0.413 * *$ & $0.509 * *$ & $0.539 * *$ & $0.405^{* *}$ & $0.534 * *$ & $0.441 * *$ \\
\hline Aortic valve (mm) & $0.442 * *$ & $0.439 * *$ & $0.448 * *$ & $0.430 * *$ & $0.403 * *$ & $0.351 * *$ & $0.413 * *$ & $0.371 * *$ \\
\hline $\begin{array}{l}\text { Sinus of Valsalva } \\
\text { diameter }(\mathrm{mm})\end{array}$ & $0.546 * *$ & $0.574 * *$ & $0.549 * *$ & $0.565 * *$ & $0.450 * *$ & $0.407 * *$ & $0.454 * *$ & $0.440 * *$ \\
\hline $\begin{array}{l}\text { Aorta annulus } \\
\text { diameter (mm) }\end{array}$ & $0.400 * *$ & $0.417 * *$ & $0.412 * *$ & $0.414 * *$ & $0.351 * *$ & $0.278^{*}$ & $0.367 * *$ & $0.322 *$ \\
\hline LVIDs (mm) & 0.004 & -0.020 & -0.002 & -0.011 & 0.131 & 0.084 & 0.123 & 0.079 \\
\hline LVIDd (mm) & 0.026 & 0.100 & 0.023 & 0.105 & 0.143 & 0.173 & 0.136 & 0.169 \\
\hline IVSDs (mm) & 0.136 & 0.250 & 0.138 & 0.236 & 0.133 & 0.188 & 0.137 & 0.188 \\
\hline IVSDd (mm) & $0.278^{*}$ & 0.216 & $0.288^{*}$ & 0.217 & 0.164 & 0.169 & 0.178 & 0.186 \\
\hline LVFWs (mm) & 0.198 & 0.251 & 0.207 & 0.241 & 0.123 & 0.170 & 0.131 & 0.213 \\
\hline LVFWd (mm) & $0.289 *$ & $0.273^{*}$ & $0.290 *$ & $0.266^{*}$ & 0.241 & 0.139 & 0.236 & 0.169 \\
\hline $\mathrm{ESV}(\mathrm{mL})$ & 0.003 & 0.076 & -0.003 & 0.076 & 0.137 & 0.162 & 0.127 & 0.152 \\
\hline $\mathrm{EDV}(\mathrm{mL})$ & 0.019 & 0.096 & 0.014 & 0.099 & 0.131 & 0.168 & 0.123 & 0.154 \\
\hline FS (\%) & -0.008 & 0.121 & -0.002 & 0.110 & -0.092 & 0.024 & -0.085 & 0.019 \\
\hline $\mathrm{EF}(\%)$ & 0.004 & 0.122 & 0.009 & 0.110 & -0.089 & 0.015 & -0.082 & 0.013 \\
\hline $\mathrm{SV}(\mathrm{mL})$ & 0.047 & 0.168 & 0.046 & 0.166 & 0.111 & 0.184 & 0.106 & 0.167 \\
\hline $\mathrm{CO}$ (L/min) & 0.050 & 0.228 & 0.040 & 0.217 & 0.011 & 0.061 & 0.001 & 0.068 \\
\hline
\end{tabular}

$\mathrm{r}=$ Pearson Correlation Coefficient

*Correlation present at $P<0.05$.

**Correlation present at $P<0.01$. 
aortic annulus diameter, sinus of Valsalva diameter, aortic root, and inconstantly with the left ventricle free wall diameter (table 3).

\section{DISCUSSION}

Cardiac ultrasound allows measuring the heart structures and the combination of B-Mode, M-Mode and Color Doppler-Mode helps to evaluate its functionality and improves the ability to diagnose and assess the severity of many forms of heart disease (Marr et al 2010). However, there is limited information concerning the echocardiographic characteristics of the RCA in horses. This is the first study that evaluates the RCA on both the short and long axis and its characteristics associated with different levels of training in Eventing horses. Fortunately, the RCA is fairly easy to visualise and evaluate during the routine cardiac ultrasound examination in long axis as previously described (Siwinska et al 2019) and in the short axis at the level of the aortic valve/ sinus of Valsalva as described in our study.

The diameter of the RCA for horses in group II was larger than the measurements obtained by Siwinska et al (2019) or the values obtained by other authors when measuring the RCA in isolated equine hearts post-mortem (Thüroff et al 1984, Gómez et al 2017). These differences can be attributed to the methodology used for measurement under different conditions; in vivo echocardiography (Siwinska et al 2019), post-mortem coronary angiography (Thüroff et al 1984) or coronary perfusion with semi-synthetic resin (Gómez et al 2017). Additionally, the type of breed used and the study design play an important role. For example, the measurements obtained by Rawling (1977) were based on 10 ponies with an average body weight of $139 \mathrm{~kg}$ while the horses analysed by Siwinska et al (2019) were horses from different breeds with a wide range of age and with a body weight ranging from 336 to $660 \mathrm{~kg}$. Differences in the echocardiographic measurements between our study and the pilot study published by Siwinska et al (2019) could be explained by the factors mentioned previously, given the positive correlation between the echocardiographic measurements and body weight (Rawlings 1977, Al-Haidar et al 2013, Siwinska et al 2019), breed (Al Haidar et al 2013) and height (Siwinska et al 2019).

To date, the vast majority of published work examining the heart's remodelling in humans and horses have focused mostly on the left ventricle (Kubo et al 1974, Young et al 2005, Iskandar and Thompson 2013, Iskandar et al 2015, Shave et al 2017). This is likely a consequence of the important role the LV plays in generating the cardiac output required to meet the demands during exercise (Shave et al 2017). Notwithstanding, cardiac adaptations to exercise/training beyond the LV have been studied and recent evidence in elite human athletes suggests that the left atrium (Iskandar et al 2015) and the aortic root diameter at the sinuses of Valsalva and aortic valve annulus respond to athletic training, nevertheless this response to training is not statistically significant and marked aortic root dilatation could likely represent a pathological process rather than a physiological adaptation to exercise (Iskandar and Thompson 2013), therefore, larger sinuses of Valsalva and aortic valve annulus diameter should be evaluated carefully by clinicians.

During training, the heart suffers a variety of physiological and structural changes as a part of the adaptation process to exercise. In human athletes and sport horses, the typical adaptations to training include lower heart rates than average, increase in end-diastolic dimension and maximal stroke volume (Crawford 1983, Bayly et al 1983). These cardiac adaptations are coupled with peripheral adaptations in skeletal muscle which increase maximal arteriovenous oxygen content difference during exercise (Bayly et al 1983, Thornton et al 1983, Tyler et al 1996). The increases in cardiac output and arteriovenous oxygen content difference during maximal exercise result in increased maximal oxygen uptake (Thornton et al 1983, Tyler et al 1996). Also, trained horses have slightly higher relative heart masses ( $1.1 \%$ of body weight) than untrained horses $(0.94 \%)$, suggesting that training causes hypertrophy of cardiac muscle (Kubo et al 1974). This hypothesis has now been supported by a number of longitudinal and cross-sectional echocardiographic studies that have demonstrated increased calculated LV mass and wall thickness following training and a decrease following detraining. Absolute and relative internal cardiac dimensions of equine athletes are also affected by race discipline (Young et al 2005).

In human athletes, whilst the existence of a specific phenotype characterised as 'athlete's heart' is generally acknowledged, the question of whether athletes exhibit characteristic vascular adaptations according to the increases in oxygen consumption by cardiac muscle, which is biologically relevant to meet the requirements during exercise, is poorly understood and that is why the concept of 'athlete's artery' as a vascular adaptation has been proposed (Green et al 2012). According to the above, there is some evidence for an impact of athletic status on arterial structure and function, so it is tempting to speculate that similar mechanisms for arterial adaptation may be at play in the equine species. In other words, it is thought that athletes have larger coronary arteries and a better vasodilation reservoir (Green et al 2012) and according to the aforementioned and the results presented in our study, the same mechanisms may be at play in the horses of our study. Clinically significant diseases of the coronary arteries in animals such as horses and dogs are rare, as opposed to what happens with humans, however, coronary artery anomalies (CAA) may lead to subsequent myocardial ischemia, which is often associated with other underlying diseases such as ventricular tachycardia, atrial fibrillation, and premature ventricular contractions (Driehuys et al 1998, Falk and Jönsson 2000, Scansen 2017) and that is why understanding coronary artery anatomy and physiology is 
essential in the study of the cardiac response to exercise and training and of other cardiovascular diseases (Cieslinski 1993, Ozgel et al 2014, Scansen 2017). Moreover, it would be also interesting to crosslink results from performance dogs and their RCA changes related to exercise with the ones in the present study.

The findings of the present work regarding the echocardiographic measurements according to the level of training/performance are very similar to those of previous reports on different types of breeds and/or discipline (Young et al 2005, Shave et al 2017). In our study, although the cardiac measurements obtained appear to be larger according to the adaptation process to training, the only measurements significantly larger were the aortic annulus diameter, sinus of Valsalva and aortic root diameter. The above can be explained by the greater pressure in the left outflow tract after systole and the capacity of the aorta to adapt to this increased pressure. On the other hand, the adaptation of the outflow tract of the left ventricle is extremely important in athletes if the entire body and musculoskeletal system have to be correctly oxygenated. Additionally, the RCA was significantly larger in horses competing at a higher level of performance in our study, which is another interesting finding that adds to the scarce information available about right coronary artery characteristics in horses. This situation has been also described in humans where athletes demonstrate increased aortic root dimensions compared with nonathlete controls (Iskandar and Thompson 2013). The aforementioned could be explained by higher oxygen demand in bigger hearts observed in trained horses, possibly due to vascular adaptation to fulfil higher cardiac muscle requirements.

Our study was not limitations-free. The study design is always a limitation when specific animals are used and outer factors need to be controlled. For example, gender distribution (21 mares versus 39 males) might have influenced the results because of the described differences in cardiac morphology between females and males in different species (Dodge et al 1992, Evans and Young 2010), nonetheless, it has been also described that dimensional echocardiographic measurements are not affected by gender in horses (Al-Haidar et al, 2013, Gomez et al 2017, Siwinska et al 2019). Also, the sample size may be low in our study $(n=60)$ but it is higher than the size used by Siwinska et al (2019) who considered only 36 horses. Moreover, in that same study, horses from different breeds, wide age range, and wide body weight were used having a variety of factors that could influence their results. In our report, a more specific and less dispersed sample of horses was selected in order to control other factors that might influence our measurements. Age bias between groups could also play an important role in the differences encountered in this study, but there is information available on the correlation between two-dimensional echocardiographic measurements and age being not significantly related in horses (Al-Haidar et al 2013, Siwinska et al 2019). Al-Haidar et al (2013) concluded that dimensional echocardiographic reference values should be established using regression equations as a function of body weight rather than age. In accordance to the above mentioned regarding the correlation between the RCA, age, height, and body weight, in our study we cannot exclude the possibility that part of the differences between groups may be due to these factors, nonetheless, the results show a positive association of RCA measurements and training/performance. Although we found a positive association between the RCA size and level of training/ performance, it will probably not be always directly related to the athlete's performance level hence further research under more controlled conditions is warranted to elucidate the clinical impact of larger RCA in performance.

In conclusion, the use of 2D echocardiography to evaluate the size and function of the heart in horses has shown low variability (Rose and Kriz 2002, Decloedt et al 2016) and it is a reliable tool to study the RCA in both short and long axis. We have established references values of the right coronary artery in a cohort of sport horses used for the eventing discipline, which complement the information already available for a more detailed cardiovascular evaluation in healthy and ill horses.

\section{REFERENCES}

Al-Haidar A, Farnir F, Deleuze S, Sandersen C, Leroux A, et al. 2013. Effect of breed, sex, age and body weight on echocardiographic measurements in the Equine species. Res Vet Sci 95, 255-260.

Arnoudse W, van Veer M, Pijls N, Woorst J, Vercauteren S, et al. 2007. Direct volumetric blood flow measurement in coronary arteries by thermodilution. J Am Coll Cardiol 50, 2294-2304.

Bayly W, Gabel A, Barr S. 1983. Cardiovascular effects of submaximal aerobic training on a treadmill in Standardbred horses, using a standardised exercise test. Am J Vet Res 44, 544-553.

Bright J, Marr C. 2010. Introduction to cardiac anatomy and physiology. In: Marr C, Bowen M (eds). Cardiology of the horse. $2^{\text {nd }}$ ed. Elsevier, Toronto, Canada, Pp 3-19.

Cieslinski G. 1993. Coronary anomalies: incidence and importance. Clin Cardiol 16, 711-715.

Crawford MH. 1992. Physiologic consequences of systematic training. Cardiol Clinics 10, 209-218.

Decloedt A, de Clercq D, Ven Sofie S, Van Der Vekens N, Sys S, et al. 2016. Echocardiographic measurements of right heart size and function in healthy horses. Equine Vet $J$ 49, 58-64.

Dodge Jr J, Brown B, Bolson E, Dodge H. 1992. Lumen diameter of normal human coronary arteries. Influence of age, sex, anatomic variation, and left ventricular hypertrophy or dilation. Circulation $86,232-246$.

Driehuys S, VanWinkle TJ, Sammarco CD, Drobatz KJ. 1998. Myocardial infarction in dogs and cats: 37 cases (1985-1994). J Am Vet Med Assoc 213, 1444-1448.

Evans D, Young L. 2010. Cardiac response to exercise and training In: Marr C, Bowen M (eds). Cardiology of the horse. $2^{\text {nd }}$ ed. Elsevier, Toronto, Canada, Pp 35-47.

Falk T, Jönsson L. 2000. Ischaemic heart disease in the dog: a review of 65 cases. J Small Anim Pract. 41, 97-103.

Fédération Equestre Internationale. 2020. Eventing Rules: $25^{\text {th }}$ Edition. Lausanne, Switzerland.

Gómez F, Ballesteros L, Estupiñan H. 2017. Morphologic expression of the right coronary artery in horses. Austral J Vet Sci 49, 161-166. 
Green D, Spence A, Rowley N, Thijseen D, Naylor L. 2012. Vascular adaptation in athletes: is there an 'athlete's artery'? Exp Physiol 97, 295-304.

Iskandar A, Thompson P. 2013. A meta-analysis of aortic root size in elite athletes exercise physiology. Circulation 127, 791-798.

Iskandar A, Mujtaba M, Thompson P. 2015. Left atrium size in elite athletes. JACC Cardiovasc Imaging 8, 753-762.

Kubo K, Senta T, Osamu, S. 1974. Relationship between training and heart in the Thoroughbred racehorse. Exp Rep Equine Hlth Lab 11, 87-93.

Kumar Raut B, Namdeo Patil V, Cherian G. Coronary artery dimensions in normal Indians. Indian Heart J 69, 512-514.

Marr C, Patteson M. 2010. Echocardiography In: Marr C, Bowen M (eds). Cardiology of the horse. $2^{\text {nd }}$ ed. Elsevier, Toronto, Canada, Pp 109-126.

Ozgel O, Haligur A, Durdun N, Karakurum E. 2014. The macroanatomy of coronary arteries in donkeys (Equus asinus L.). Anat Histol Embryol 33, 278-283.

Rawlings C. 1977. Coronary arterial anatomy of the small pony. Am J Vet Res 38, 1031-1035.

Rose R, Kriz N. 2008. Repeatability of standard transthoracic echocardiographic measurements in horses. Aust Vet J 80, 362-370

Scansen B. 2017. Coronary artery anomalies in animals. Vet Sci 4, 20.

Shave R, Howatson G, Dickson D, Young L. 2017. Exercise-induced cardiac remodeling: Lessons from humans, horses, and dogs. Vet Sci 4, 9.
Siwinska N, Michalek M, Zak A, Slowikowska M, Noszczyk-Nowak A, et al. 2019. Two-dimensional echocardiographic measurements of the right coronary artery in healthy horses. BMC Vet Res 15, 43.

Thornton J, Essen-Gustavsson B, Lindhohn A, McMiken D, Persson S, et al. 1983. Effects of training and detraining on oxygen uptake, cardiac output, blood gas tensions, $\mathrm{pH}$ and lactate concentrations during and after exercise in the horse. In: Snow DH, Persson SGB, Rose RJ (eds). Equine Exercise Physiology. Granta Editions, Cambridge, UK, Pp 470-486.

Thüroff J, Hort W, Lichti H. 1984. Diameter of coronary arteries in 36 species of mammalian from mouse to giraffe. Basic Res Cardio 179, 199-206.

Trachsel D, Giraudet A, Maso D, Hervé G, Hauri D, et al. 2016. Relationships between body dimensions, body weight, age, gender, breed and echocardiographic dimensions in young endurance horses. BMC Vet Res 12, 226.

Tyler C, Golland L, Evans D, Hodgson D, Rose R. 1996. Changes in maximum oxygen uptake during prolonged training, overtraining, and detraining in horses. J Appl Physiol 81, 2244-2249.

Young LE, Rogers K, Wood JL. 2005. Left ventricular size and systolic function in Thoroughbred racehorses and their relationships to race performance. J Appl Physiol 99, 1278-1285. 
